Canadian Science Publishing

Biochemistry and Cell Biology

Biochimie et biologie cellulaire

\title{
Cost-effective elimination of lipofuscin fluorescence from formalin-fixed brain tissue by white phosphor light emitting diode array
}

\begin{tabular}{|r|l|}
\hline Journal: & Biochemistry and Cell Biology \\
\hline Manuscript ID & bcb-2016-0125 \\
\hline Manuscript Type: & Methods \\
\hline Date Submitted by the Author: & 08 -Jun-2016 \\
\hline Complete List of Authors: & $\begin{array}{l}\text { Sun, Yulong; University of Toronto, Medical Biophysics } \\
\text { Chakrabartty, Avi; University of Toronto, Medical Biophysics; University of } \\
\text { Toronto, Biochemistry }\end{array}$ \\
\hline Keyword: & $\begin{array}{l}\text { autofluorescence, lipofuscin, photobleaching, light emitting diode, } \\
\text { fluorescence microcopy }\end{array}$ \\
\hline
\end{tabular}

\section{SCHOLARONE ${ }^{\text {m }}$ \\ Manuscripts}


1 Cost-effective elimination of lipofuscin fluorescence from formalin-fixed brain tissue by

2 white phosphor light emitting diode array

3 Yulong Sun $^{1, *}$ and Avi Chakrabartty ${ }^{1,2}$

$4{ }^{1}$ Department of Medical Biophysics, Princess Margaret Cancer Centre, University of Toronto, 5 Toronto, Canada

$6{ }^{2}$ Department of Biochemistry, Princess Margaret Cancer Centre, University of Toronto, Toronto, 7 Canada

8 *to whom correspondence should be addressed.

9 Princess Margret Cancer Research Tower, 101 College Street, Room 4-205, Toronto, ON,

10 M5G1L7, Canada. E-mail: yulong.sun@mail.utoronto.ca 


\section{ABSTRACT:}

12 Autofluorescence of aldehyde-fixed tissues greatly hinders fluorescence microscopy. In

13 particular, lipofuscin, an autofluorescent component of aged brain tissue, complicates

14 fluorescence imaging of tissue in neurodegenerative diseases. Background and lipofuscin

15 fluorescence can be reduced by greater than $90 \%$ through photobleaching using white phosphor

16 light emitting diode arrays prior to treatment with fluorescent probes. We compared the effect of

17 photobleaching versus established chemical quenchers on the quality of fluorescent staining in

18 formalin-fixed brain tissue of frontotemporal dementia with tau-positive inclusions. Unlike

19 chemical quenchers, which reduced fluorescent probe signals as well as background,

20 photobleaching treatment had no effect on probe fluorescence intensity while it effectively

21 reduced background and lipofuscin fluorescence. The advantages and versatility of

22 photobleaching over established methods are discussed.

23 Keywords: autofluorescence; lipofuscin; photobleaching; light emitting diode; fluorescence

24 microcopy 


\section{INTRODUCTION:}

26 The successful visualization of fluorescent markers in tissue is often complicated by

27 autofluorescence. Many sources of endogenous autofluorescence exist in mammalian tissues,

28 such as lipofuscin, collagen, and elastin (Banerjee et al. 1999, Ottis et al. 2012). Formalin

29 fixation, a common method of sample preparation, also generates autofluorescence. Lipofuscin,

30 whose presence is a common hallmark of aging, is a particular complication in fluorescence

31 imaging of specimens of neurodegerative diseases, where patients are often of advanced age

32 (Ottis et al. 2012). Lipofuscin's broad fluorescence emission greatly hinders the application of

33 common fluorescent labels like FITC and Texas Red. Lipofuscin signals often need to be

34 manually labeled in fluorescence images to aid image interpretation (Xiao et al. 2015).

35 A number of treatment options have been suggested to reduce lipofuscin fluorescence. These

36 include chemical treatment of the specimen with $\mathrm{CuSO}_{4}$ in ammonium acetate or quenching dyes

37 such as Sudan Black B and Eriochrome Black T (Davis et al. 2014). Digital image processing by

38 spectral un-mixing has also been used, but the process is difficult and has the disadvantage of

39 generating false positives and artifacts (Zimmermann et al. 2003). Previously, photobleaching

40 using a fluorescent tube lamp has been performed, although certain lipofuscin structures still

41 remain unaffected after 48 hours in brain tumour sections (Neumann and Gabel 2002). It has also

42 been reported previously that RGB-based, multispectral light emitting diode (LED) arrays are

43 effective at reducing autofluorescence, though the apparatus may be cost-prohibitive (Duong and

44 Han 2013). The most thorough photobleaching can be achieved by irradiating the sample under a

45 fluorescence microscope using a high powered laser source through an objective (Schnell et al.

46 1999, Neumann and Gabel 2002). However, this method is impractical due to the limited field of 
47 view of the objective compared to the size of the sample. Prolonged exposure will also cause

48 sample damage.

49 Recently, white phosphor LEDs used in household lighting have emerged as an alternative light

50 source for fluorescence microscopy over halogen lamps due to their wide emission spectrum,

51 high luminosity, and very low manufacturing cost (Albeanu et al. 2008, Robertson et al. 2009).

52 Phosphor-based LEDs generate white light through a single blue LED surrounded by yellow

53 phosphors, and are less costly than their RGB-based counterparts. Given the viability of white

54 LEDs as a fluorescence excitation source, we propose a simple, effective, and widely applicable

55 method to reduce autofluorescence from formalin-fixed tissue by photobleaching the specimen

56 using a commercially available LED lamp prior to staining and immunofluorescence imaging. In

57 the study by Duong and Han, an apparatus was constructed using custom scaffolds, slide holders,

58 and an array of high power multispectral LEDs in an enclosed refrigeration unit. The cost of raw

59 materials, fabrication and construction for this apparatus may be upwards of 1000 USD. In the

60 present study, we show that an equivalent result can be achieved using off-the-shelf components

61 for less than $1 \%$ of the cost.

62 We quantified the efficacy of white phosphor LED photobleaching in formalin-fixed human

63 brain tissue from a patient with frontotemporal lobar degeneration with tau-positive inclusions

64 (FTLD-T). We stained the tissue with a number of fluorescent probes and compared and

65 quantified the quality of staining in sections that have been pre-treated with photobleaching or

66 treated with chemical quenchers TrueBlack ${ }^{\mathrm{TM}}$ (a proprietary formula) or Eriochrome Black T.

67 We demonstrate that photobleaching, unlike chemical quenchers, does not suppress fluorescent

68 probe signals. 


\section{MATERIALS AND METHODS:}

\section{Photobleaching apparatus}

A 6 watt, 800 lux LED array desk lamp with flexible arm and flat lighting surface (DBpower, Shenzhen, Guangdong, China) was inverted such that the lamp could be placed under a square petri dish (Sarstedt, Nümbrecht, Germany) containing sterile buffer. The dish was elevated by a plastic support to avoid sample heating (Figure 1A). Samples on microscope slides were photobleached by submerging the slides in sterile TBS and $0.05 \%$ sodium azide at $4{ }^{\circ} \mathrm{C}$ in a cold room. A reflective dome covered the petri dish containing the sample for the duration of photobleaching. The emission spectrum $(400-800 \mathrm{~nm})$ of the lamp was measured using a QM-1 fluorescence spectrophotometer (Photon Technology International, Edison, NJ, USA).

\section{Sample preparation and Immunofluorescence}

Formalin-fixed brain blocks of the orbitofrontoal gyri in a case of FTLD-T ( 2 days fixation) were run through $10 \%, 20 \%$, and $30 \%$ sucrose gradients, infiltrated with optimal cutting temperature (OCT) compound, frozen, and cut into $10 \mu \mathrm{m}$ thick sections and attached to glass microscope slides. For samples undergoing photobleaching treatment, slides were photobleached for 48 hours in the photobleaching apparatus prior to staining. The slides were treated in antigen retrieval buffer (10 mM Citric Acid, 2 mM EDTA, $0.05 \%$ Tween 20, pH 6.2) for 30 minutes at $90{ }^{\circ} \mathrm{C}$, then washed twice for 5 minutes in TBS plus $0.025 \%$ Triton X-100 (TBS-Triton). The slides were blocked in 10\% normal goat serum (Aurion, Wageningen, The Netherlands) and 1\% BSA (ThermoFisher Scientific, Waltham, MA, USA) in TBS-Triton for 2 hours at room temperature. Primary mouse anti-phospho-PHF-tau pSer202+Thr205 (AT8) antibody (1:50, ThermoFisher Scientific, Waltham, MA, USA) in $1 \%$ BSA/TBS was applied overnight at $4{ }^{\circ} \mathrm{C}$ in 
91 a humidified chamber. The slides were rinsed $2 \times 5$ min with TBS-Triton and a mix of secondary

92 antibodies consisting of Alexa 488 goat anti-mouse (1:100, ThermoFisher Scientific, Waltham,

93 MA, USA) and Texas red goat anti-mouse (1:100, ThermoFisher Scientific, Waltham, MA,

94 USA) in 1\% BSA/TBS was applied for 1 hour at room temperature in the dark. The slides were

95 rinsed $2 \times 5 \mathrm{~min}$ in TBS and stained with DAPI $(0.25 \mu \mathrm{g} / \mathrm{mL}$, ThermoFisher Scientific,

96 Waltham, MA, USA) for 10 minutes. Slides not receiving photobleaching treatment were then

97 either treated with TrueBlack ${ }^{\mathrm{TM}}$ lipofuscin autofluorescence quencher (Biotium, Hayward, CA,

98 USA) according to manufacturer's instructions or with 1.65\% Eriochrome Black T (Sigma-

99 Aldrich, St. Louis, MO, USA) in $\mathrm{ddH}_{2} \mathrm{O}$ for 5 minutes according to established protocols (Davis

100 et al. 2014). The slides were rinsed $3 \times 5 \mathrm{~min}$ in TBS to remove excess quencher and mounted

101 with Immu-mount aqueous mounting medium (ThermoFisher Scientific, Waltham, MA, USA)

102 for fluorescence microscopy. For Nissl staining, the above protocol was altered such that the

103 blocking and antibody diluting buffers contained $0.5 \%$ fish gelatin (Electron Microscopy

104 Sciences, Hatfield, PA, USA) in TBS instead of BSA or serum, and only Alexa 488 conjugated

105 antibody was used for secondary antibody staining. Following DAPI staining and prior to

106 mounting, NeuroTrace ${ }^{\circledR} 530 / 615$ red fluorescent Nissl stain (ThermoFisher Scientific, Waltham,

107 MA, USA) was applied to the slides according to manufacturer's instructions.

108 Fluorescence microscopy and image quantitation

109 For detection of autofluorescence, several unstained sections were photobleached using the

110 photobleaching apparatus for up to 72 hours. At various time points, the slides were removed

111 from the photobleaching apparatus, coverslipped in sterile TBS and imaged using a Zeiss LSM

112710 confocal microscope (Zeiss, Oberkochen, Germany) with a Plan Apochromat 10× 0.45 NA

113 objective (Zeiss, Oberkochen, Germany). For the Alexa 488 channel, the sample was excited at 
$114488 \mathrm{~nm}$ (argon laser) and detected at 493-570 nm. For the Texas Red channel, the sample was

115 excited at $561 \mathrm{~nm}$ (DPSS $561 \mathrm{~nm}$ laser) and detected at 601-635 nm. For stained slides,

116 fluorescence at Alexa $488\left(\lambda_{\mathrm{ex}}=488 \mathrm{~nm}, \lambda_{\mathrm{em}}=493-605 \mathrm{~nm}\right)$, Texas Red $/ \mathrm{Nissl}\left(\lambda_{\mathrm{ex}}=561 \mathrm{~nm}, \lambda_{\mathrm{em}}\right.$

$117=566-689 \mathrm{~nm})$, and DAPI $\left(\lambda_{\mathrm{ex}}=405 \mathrm{~nm}\right.$; Diode 405 laser, $\left.\lambda_{\mathrm{em}}=410-507 \mathrm{~nm}\right)$ channels were

118 measured. Laser power and gain settings were kept identical using Zen software for each set of

119 comparative images. Lipofuscin fluorescence was quantified by particle analysis in ImageJ

120 (Schneider et al. 2012), plotted, and fitted to a single exponential decay function $y=y_{0}+A e^{\frac{-x}{T}}$

121 using Origin 8.5 software (OriginLab, Northampton, MA, USA), where $y$ is the intensity, $\mathrm{x}$ is the

122 photobleaching duration and $\mathrm{T}$ is the decay constant. Fluorescence RGB profiling was measured

123 using ImageJ and the 'RGBProfiler' ImageJ plugin (http://rsb.info.nih.gov/ij/plugins/rgb-

124 profiler.html). 


\section{RESULTS AND DISCUSSION:}

126

127 Autofluorescence of unstained brain tissue from a case of FLTD-T was measured before

128 photobleaching and at 12 hour intervals of LED photobleaching. The emission peaks of the lamp

129 at $450 \mathrm{~nm}$ and 500-600 $\mathrm{nm}$ suggests that it can effectively target the excitation wavelengths of a

130 broad range of visible-light chromophores (Figure 1B). While previous studies targeted

131 individual emission peaks with multispectral LEDs (Duong and Han 2013), we found that white

132 phosphor LEDs could produce a similar emission spectrum. It was evident from the photographs

133 of the sections that the majority of background fluorescence was eliminated after 24 hours

134 (Figure 1C), while the fluorescent, lipofuscin-like speckles were nearly all photobleached after

13572 hours. Using the exponential decay function for quantification, we calculated the half-life of

136 fluorescent intensity to be 4.6 and 7.1 hours for Alexa 488 and Texas Red channels respectively

137 (Figure 2), however, particles with reduced intensities were still generally visible in the

138 photographs at this stage (Figure 1C). Using the decay constants, we calculated that 95\% of the

139 fluorescence should be removed after 20 and 31 hours for the Alexa 488 and Texas Red channels

140 respectively. Visually, we found that after 36 hour photobleaching, the intensities of lipofuscin

141 particles became sufficiently low compared to intended fluorescent signals, allowing for

142 generation of immunofluorescence images with little to no background. We used 48 hours as a

143 convenient and effective photobleaching duration for subsequent experiments.

\section{Immunostaining of tau-positive inclusions}

145 Immunostaining for hyperphosphorylated tau was performed to visualize pathological tau

146 inclusions in the FTLD-T case (Figure 3). We tested the efficacy of 48 hour photobleaching (PB) 
147 compared to a commercially available lipofuscin autofluorescence quencher TrueBlack ${ }^{\mathrm{TM}}(\mathrm{TB})$

148 and a previously reported treatment protocol using Eriochrome Black T (EBT) (Davis et al.

149 2014). The tissues were stained with AT8 antibody, followed by commonly used Alexa 488- and

150 Texas Red-conjugated fluorescent secondary antibodies and counterstained with the nuclear stain

151 DAPI. In the untreated sample, autofluorescence was ubiquitous in both the Texas Red and

152 Alexa 488 channels, significantly compromising the quality of the image. Signal profiling of the

153 image showed that the tau inclusions were stained with relatively high intensity for both

154 secondary antibodies. However, the lipofuscin intensity in the Texas Red channel was

155 comparable to the intensity of the Texas Red secondary fluorescence, leading to complications in

156 image interpretation. EBT-treated samples showed over-quenching and complete loss of the

157 DAPI signal. The dye also generated an undesirable basal level of fluorescence in the Texas Red

158 channel. EBT appeared to be effective in reducing Alexa 488 channel autofluorescence, but

159 indiscriminately reduced the secondary antibody fluorescence as well, limiting its utility. Both 48

160 hour PB and TB treatment produced images with minimal background florescence. However, in

161 the 48 hour PB treatment, intensities of tau inclusions labeled by Alexa 488 and Texas Red

162 fluorescence were relatively similar based on their signal profiles, whereas the samples treated

163 with TB had significantly reduced Texas Red and DAPI signals.

164 We then investigated the effect of PB and TB treatments on more sensitive stains such as Nissl,

165 which can be quenched by BSA and other serum proteins (Figure 4). We stained the tissue with

166 AT8 primary antibody followed by Alexa 488 secondary as before and applied NeuroTrace ${ }^{\circledR}$

$167515 / 630$ Nissl stain which labeled neurons within the tissue. The specimen was counterstained

168 with DAPI. Similar to our previous results, PB treatment did not appear to weaken the

169 fluorescence of the Nissl stain in the 570-690 nm emission range, and the cell bodies of the 
170 neurons were readily visible and distinct from the DAPI nuclear staining. In contrast, the TB

171 treated samples showed reduced Nissl staining which largely overlapped with the DAPI signal

172 (Figure 4). It is possible that the TB treatment quenched the weaker staining of Nissl bodies in

173 the endoplasmic reticulum, but did not penetrate into the nucleus, which Nissl also stains (Kádár

174 et al. 2009).

175 Overall, we find that irradiation of formalin-fixed brain tissue with a white phosphor LED lamp

176 is an effective and low-cost method of reducing autofluorescence. Photobleaching does not

177 interfere with the fluorescence intensity of probes due to the fact that it can be applied prior to

178 sample staining. This allows for exclusive bleaching of unwanted autofluorescence, whereas

179 commercial dyes and quenchers such as TrueBlack ${ }^{\mathrm{TM}}$ are applied prior to mounting the sample,

180 allowing for undesirable quenching of probe signals. Additionally, photobleaching does not

181 introduce any exogenous material to the specimen that potentially interferes with subsequent

182 handling. Quenchers such as TrueBlack ${ }^{\mathrm{TM}}$ must also be applied in $70 \%$ ethanol. Compared to the

183 commercial quencher TrueBlack ${ }^{\mathrm{TM}}$, our photobleaching protocol, which uses a commercial lamp,

184 requires more processing time. Our LED array is also not expected to photobleach fluorescence

185 excitable near UV range since the lamp has no emission at wavelengths below $405 \mathrm{~nm}$.

186 However, LED with higher power and intensity can reduce the processing time, and as previous

187 studies have described, custom LEDs with different emission wavelengths can be used to direct

188 photobleaching to specific chromophores (Duong and Han 2013). For targeting broad-spectrum

189 autofluorescence, however, it appears that white phosphor LEDs is sufficiently effective.

190 We demonstrate that photobleaching treatment followed by immunostaining generally produces

191 signals that are brighter and more intense at certain wavelengths compared to post-staining

192 autofluorescence suppression approaches, while producing little to no background. The 
193 generation of clean images for immunofluorescence microscopy is not only important for the

194 accurate visualization of features of interest, but it is also a prerequisite for the application of

195 advanced microscopy techniques such as spatially targeted optical micro-proteomics (STOMP)

196 (Hadley et al. 2015). While previous methods have involved intricately assemblies, we present

197 an extremely simple, inexpensive, but effective method for autofluorescence removal that is

198 accessible to all investigators. Overall, photobleaching using white phosphor LED prior to

199 staining is a versatile treatment that we expect to be amiable to a broad range of specimens.

\section{AUTHOR CONTRIBUTIONS:}

201 Y. S contributed to experimental design, conducted experiments, and analyzed data. A. C.

202 conceived the study. All authors participated in the drafting of the manuscript.

\section{ACKNOWLEDGEMENTS:}

204 This study was supported by the Canadian Consortium of Neurodegeneration and Aging

205 (CCNA). The authors would like to thank Sultan Darvesh, and Andrew Reid from the Maritime

206 Brain Tissue Bank for providing the FTLD brain tissues. Milan Ganguly from Spatio-temporal

207 Targeting and Amplification of Radiation Response (STTARR) imaging center is thanked for

208 preparing and sectioning the tissues. We also thank Kevin C. Hadley for helpful discussions and

209 editing the manuscript.

\section{ETHICS:}

211 The work presented was performed in compliance with recognized international standards,

212 including the International Conference on Harmonization (ICH), the Council for International

213 Organizations of Medical Sciences (CIOMS) and the principles of the Declaration of Helsinki.

214 Use of human tissue was in accordance with the University Health Network Research Ethic 
215 Board. The human brain samples were collected as a part of the Maritime Brain Tissue Bank. At

216 the time of collection, informed consent was obtained.

\section{COMPETING INTERESTS:}

218 The authors declare no competing interests. 


\section{REFERENCES:}

220 Albeanu, D.F., Soucy, E., Sato, T.F., Meister, M., and Murthy, V.N. 2008. LED arrays as cost 221 effective and efficient light sources for widefield microscopy. PLoS One 3(5): 1-7.

222 Banerjee, B., Miedema, B.E., and Chandrasekhar, H.R. 1999. Role of basement membrane 223 collagen and elastin in the autofluorescence spectra of the colon. J. Investig. Med. 47(6): 326-32.

225

226

227

228

229

230

231

232

233

234

235

236

237

238

239

240

Davis, A.S., Richter, A., Becker, S., Moyer, J.E., Sandouk, A., Skinner, J., and Taubenberger, J.K. 2014. Characterizing and Diminishing Autofluorescence in Formalin-fixed Paraffinembedded Human Respiratory Tissue. J. Histochem. Cytochem. 62(6): 405-423.

Duong, H., and Han, M. 2013. A multispectral LED array for the reduction of background autofluorescence in brain tissue. J. Neurosci. Methods 220(1): 46-54. Elsevier B.V.

Hadley, K.C., Rakhit, R., Guo, H., Sun, Y., Jonkman, J.E., McLaurin, J., Hazrati, L.-N., Emili, A., and Chakrabartty, A. 2015. Determining composition of micron-scale protein deposits in neurodegenerative disease by spatially targeted optical microproteomics. Elife 4: 1-21.

Kádár, A., Wittmann, G., Liposits, Z., and Fekete, C. 2009. Improved method for combination of immunocytochemistry and Nissl staining. J. Neurosci. Methods 184(1): 115-118.

Neumann, M., and Gabel, D. 2002. Simple method for reduction of autofluorescence in fluorescence microscopy. J. Histochem. Cytochem. 50(3): 437-9.

Ottis, P., Koppe, K., Onisko, B., Dynin, I., Arzberger, T., Kretzschmar, H., Requena, J.R., Silva, C.J., Huston, J.P., and Korth, C. 2012. Human and rat brain lipofuscin proteome. Proteomics 12(15-16): 2445-2454.

Robertson, J.B., Zhang, Y., and Johnson, C.H. 2009. Light-emitting diode flashlights as effective 

and inexpensive light sources for fluorescence microscopy. J. Microsc. 236(1): 1-4.

242 Schneider, C. a, Rasband, W.S., and Eliceiri, K.W. 2012. NIH Image to ImageJ: 25 years of 243 image analysis. Nat. Methods 9(7): 671-675. Nature Publishing Group.

244 Schnell, S.A., Staines, W.A., and Wessendorf, M.W. 1999. Reduction of Lipofuscin-like 245 Autofluorescence in Fluorescently Labeled Tissue. J. Histochem. Cytochem. 47(6): 719246730.

247 Xiao, S., Sanelli, T., Chiang, H., Sun, Y., Chakrabartty, A., Keith, J., Rogaeva, E., Zinman, L., 248 and Robertson, J. 2015. Low molecular weight species of TDP-43 generated by abnormal 249 splicing form inclusions in amyotrophic lateral sclerosis and result in motor neuron death. $250 \quad$ Acta Neuropathol. 130(1): 49-61.

251 Zimmermann, T., Rietdorf, J., and Pepperkok, R. 2003. Spectral imaging and its applications in 252 live cell microscopy. FEBS Lett. 546(1): 87-92. 
254 Figure 1: Time-dependent photobleaching of formalin-fixed brain tissue using a white

255 phosphor LED array. A) Photograph of photobleaching apparatus indicating the LED array

256 light source, plastic platform, and sample chamber where a mock slide is placed in buffer. A

257 reflective dome is constructed using aluminum foil to cover the apparatus during photobleaching.

258 B) Emission spectrum of LED array consists of a sharp peak at $450 \mathrm{~nm}$ and a broad peak

259 centered at $550 \mathrm{~nm}$ and spanning 500-600 nm (arbitrary fluorescence units). No significant

260 emission below $400 \mathrm{~nm}$ or above $650 \mathrm{~nm}$ is observed. C) Photographs of 2 fields of view of

261 unstained $10 \mu \mathrm{m}$ thick brain section of grey matter in a case of FTLD-T imaged at Alexa $488\left(\lambda_{\mathrm{ex}}\right.$

$\left.262=488 \mathrm{~nm}, \lambda_{\mathrm{em}}=493-570 \mathrm{~nm}\right)$ and Texas Red $\left(\lambda_{\mathrm{ex}}=561 \mathrm{~nm}, \lambda_{\mathrm{em}}=601-635 \mathrm{~nm}\right)$ channels 0 to 72

263 hours after photobleaching. Distinct fluorescent particles resembling lipofuscin are observed and

264 their signal intensities are reduced after 72 hours of photobleaching, although a few dim

265 fluorescent bodies remain. Scale bar $=100 \mu \mathrm{m}$.

Figure 2: Quantification of LED-induced signal intensity reduction of lipofuscin

267 fluorescence in two fields of view. Each dot represents the signal intensity of one lipofuscin

268 particle present in a field of view at Alexa 488 (left) and Texas Red (middle) channels. Particles

269 from two $425 \mu \mathrm{m} \times 425 \mu \mathrm{m}$ fields of view are quantified and plotted. The population of

270 fluorescent particles shifts to reduced intensity levels after photobleaching. The mean and

271 standard deviation of the population is plotted (right) and fitted to the exponential decay function

$272 y=y_{0}+A e^{\frac{-x}{T}}$, where $\mathrm{y}$ is the intensity, $\mathrm{y}_{0}$ is the baseline intensity, $\mathrm{A}$ is the initial maximum

273 intensity, $\mathrm{x}$ is the photobleaching duration, and $\mathrm{T}$ is the decay constant. The fitted parameters for

274 each channel is listed under their figure legends. 
275 Figure 3: Immunofluorescence imaging of phospho-tau stained FTLD-T formalin-fixed

276 brain tissue. $10 \mu \mathrm{m}$ thick sections were stained with AT8 primary antibody and Texas Red and

277 Alexa 488 conjugated secondary antibodies. Slides were counterstained with DAPI. The signals

278 from Alexa $488\left(\lambda_{\mathrm{ex}}=488 \mathrm{~nm}, \lambda_{\mathrm{em}}=493-570 \mathrm{~nm}\right)$, Texas Red $\left(\lambda_{\mathrm{ex}}=561 \mathrm{~nm}, \lambda_{\mathrm{em}}=601-635\right.$

$279 \mathrm{~nm}), \mathrm{DAPI}\left(\lambda_{\mathrm{ex}}=405 \mathrm{~nm}, \lambda_{\mathrm{em}}=410-507 \mathrm{~nm}\right)$ and a merged image are displayed. Multiple

280 autofluorescence reduction methods including 48 hour photobleaching, TrueBlack ${ }^{\mathrm{TM}}$,

281 Eriochrome Black T, and non-treatment are tested. Signal profiles at the cross section indicated

282 by the dotted lines on the merged panels are quantified. Signal peaks representing Tau inclusions

283 (T), nuclei $(\mathrm{N})$ or lipofuscin particles $(\mathrm{L})$ are labeled. Scale bar $=100 \mu \mathrm{m}$.

284 Figure 4: Immunofluorescence imaging of phospho-tau and Nissl stained FTLD-T formalin

285 fixed brain tissue using photobleaching and TrueBlack ${ }^{\text {TM }}$ treatments. $10 \mu \mathrm{m}$ thick sections

286 were stained with AT8 primary antibody and Alexa 488 conjugated secondary antibody,

287 NeuroTrace ${ }^{\circledR}$ fluorescent Nissl stain, and DAPI. The signals from Alexa $488\left(\lambda_{\mathrm{ex}}=488 \mathrm{~nm}, \lambda_{\mathrm{em}}\right.$

$288=493-570 \mathrm{~nm}), \operatorname{Nissl}\left(\lambda_{\mathrm{ex}}=561 \mathrm{~nm}, \lambda_{\mathrm{em}}=601-635 \mathrm{~nm}\right)$, DAPI $\left(\lambda_{\mathrm{ex}}=405 \mathrm{~nm}, \lambda_{\mathrm{em}}=410-507\right.$

$289 \mathrm{~nm}$ ) and a composite channel are displayed. The sections are either photobleached prior to

290 staining, or treated with TrueBlack ${ }^{\mathrm{TM}}$ after staining. Scale bar $=100 \mu \mathrm{m}$. 
A

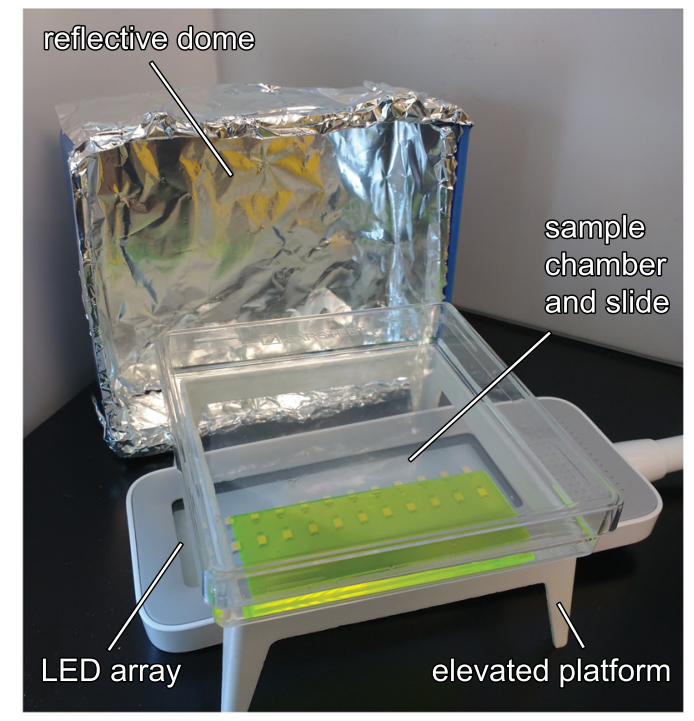

C

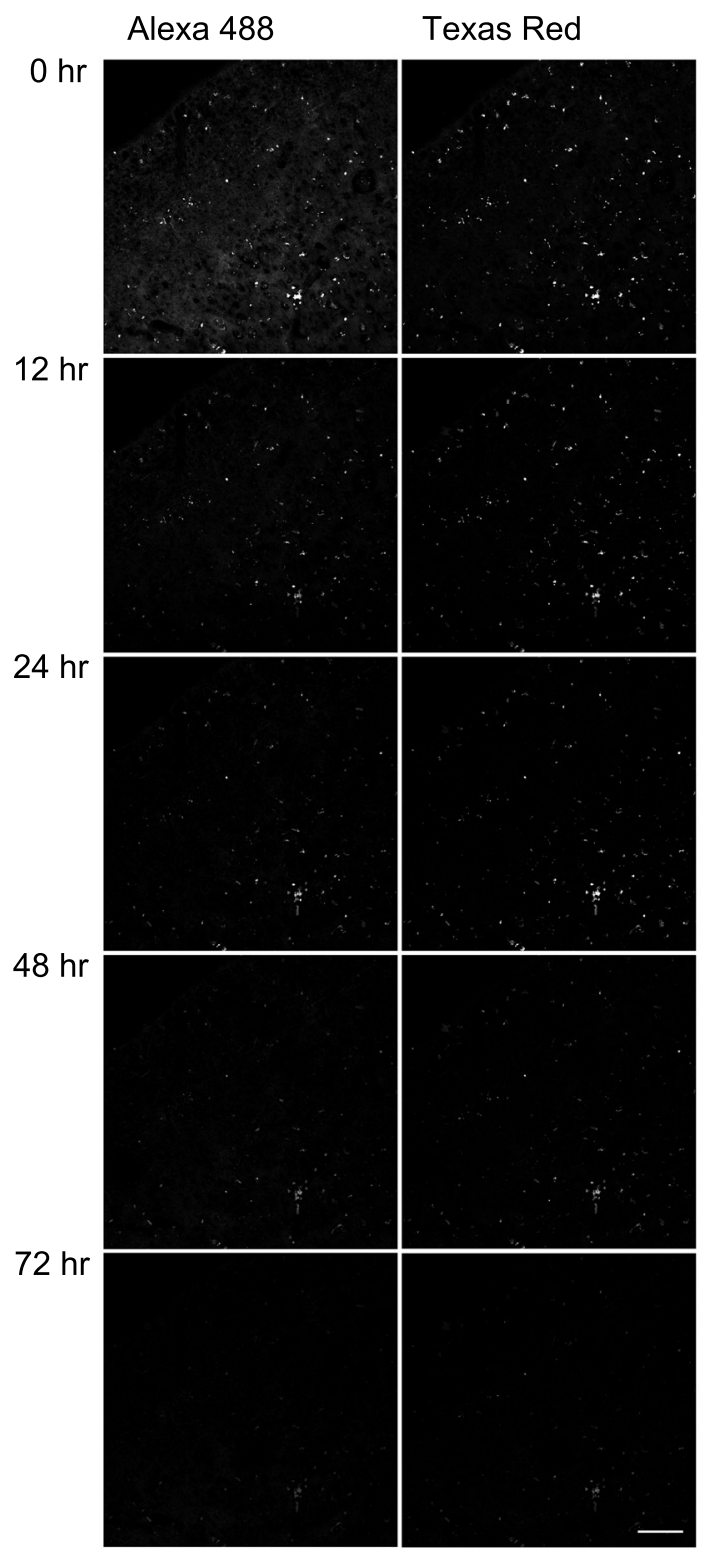

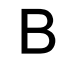

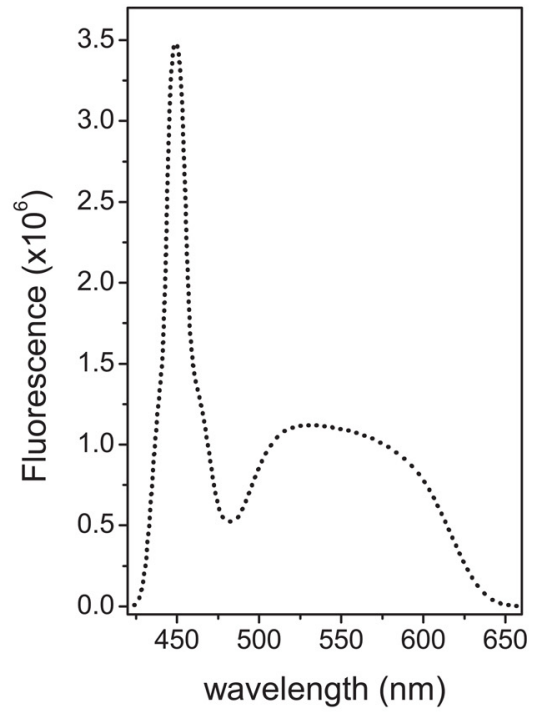

Field of view 2

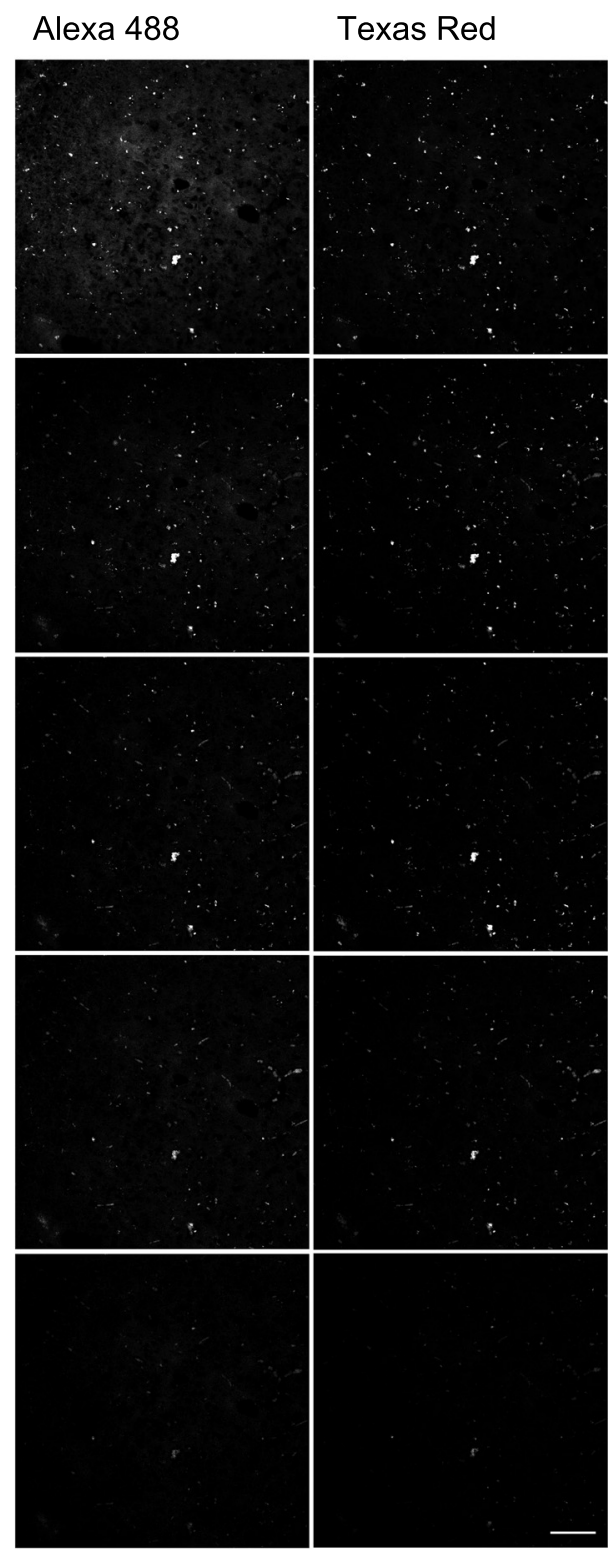

https://mc06.manuscriptcentral..com/bcb-pubs 
Biochemistry and Cell Biology

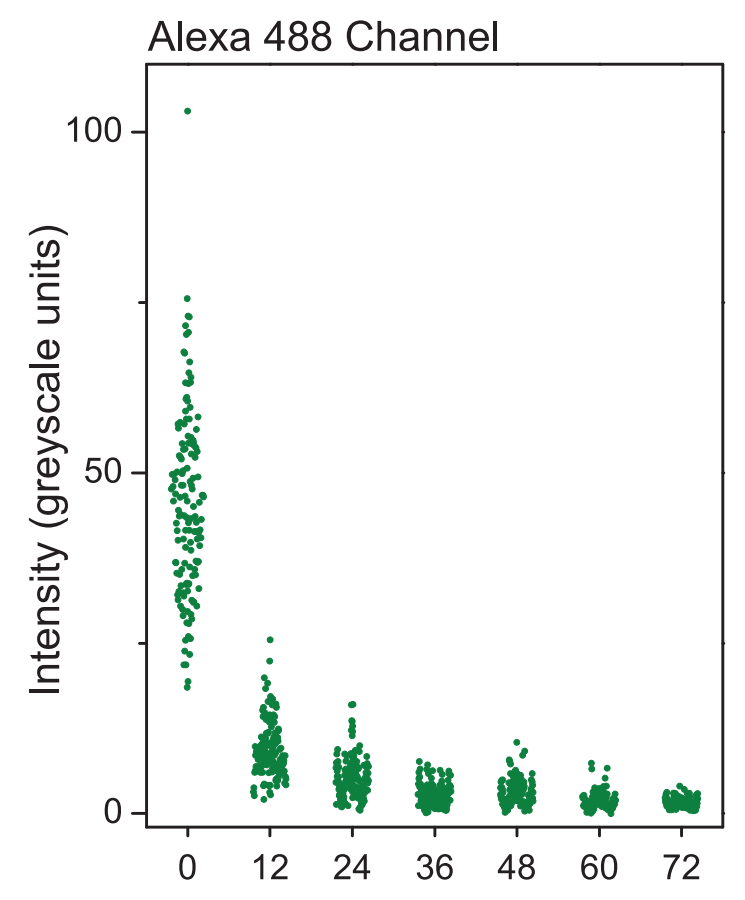

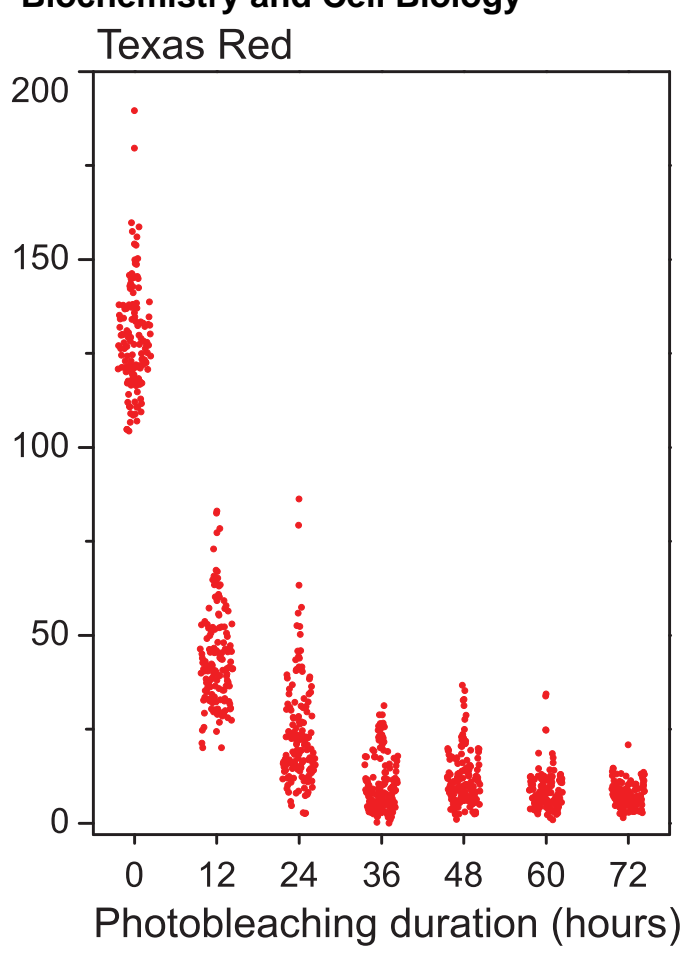

Page 18 of 20 Exponential Decay Curves

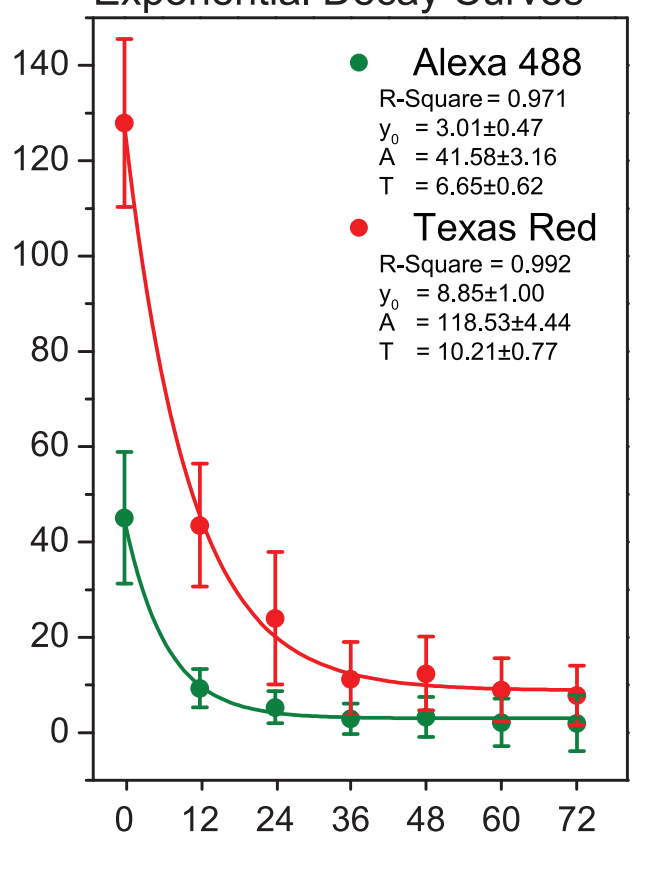




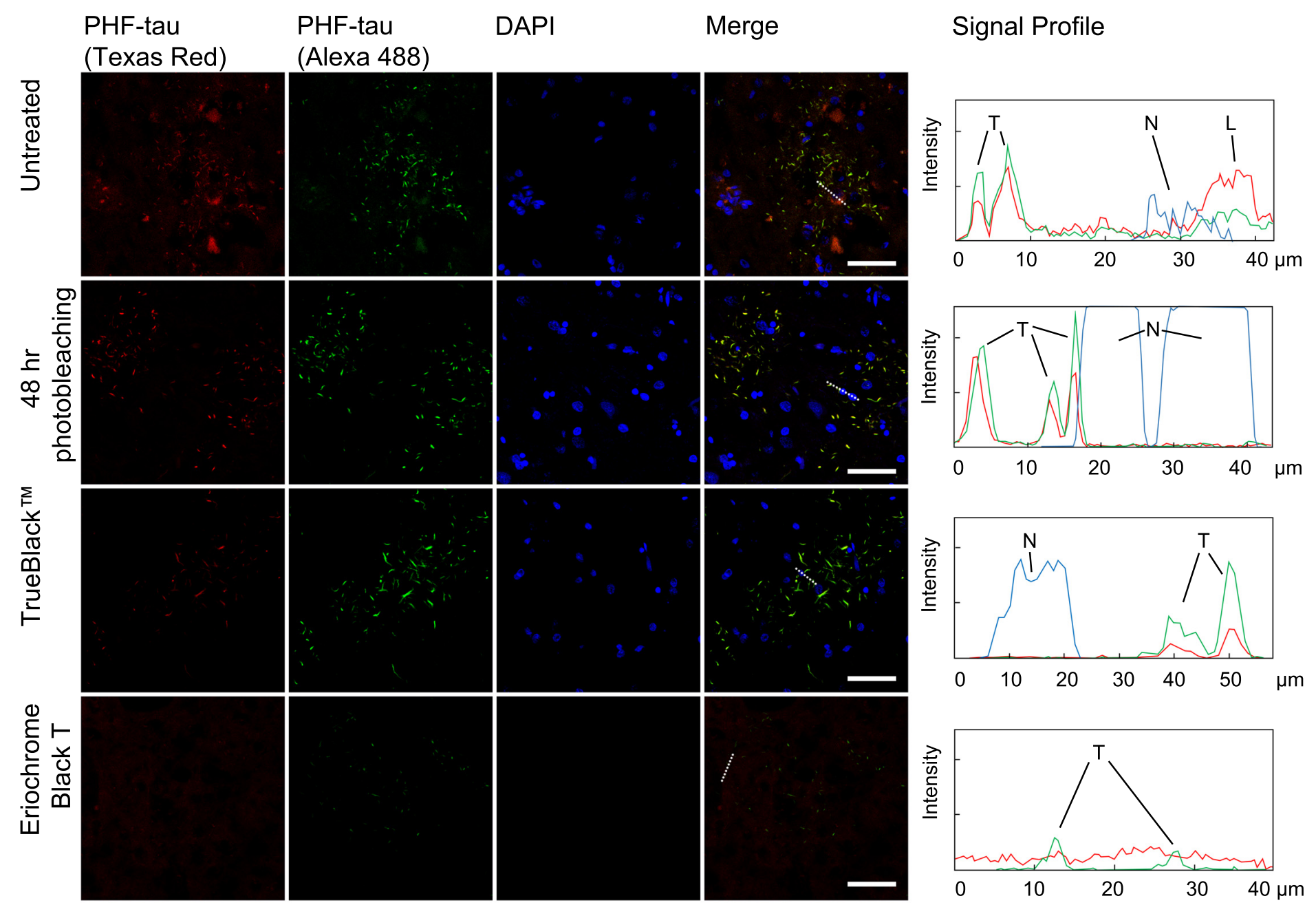




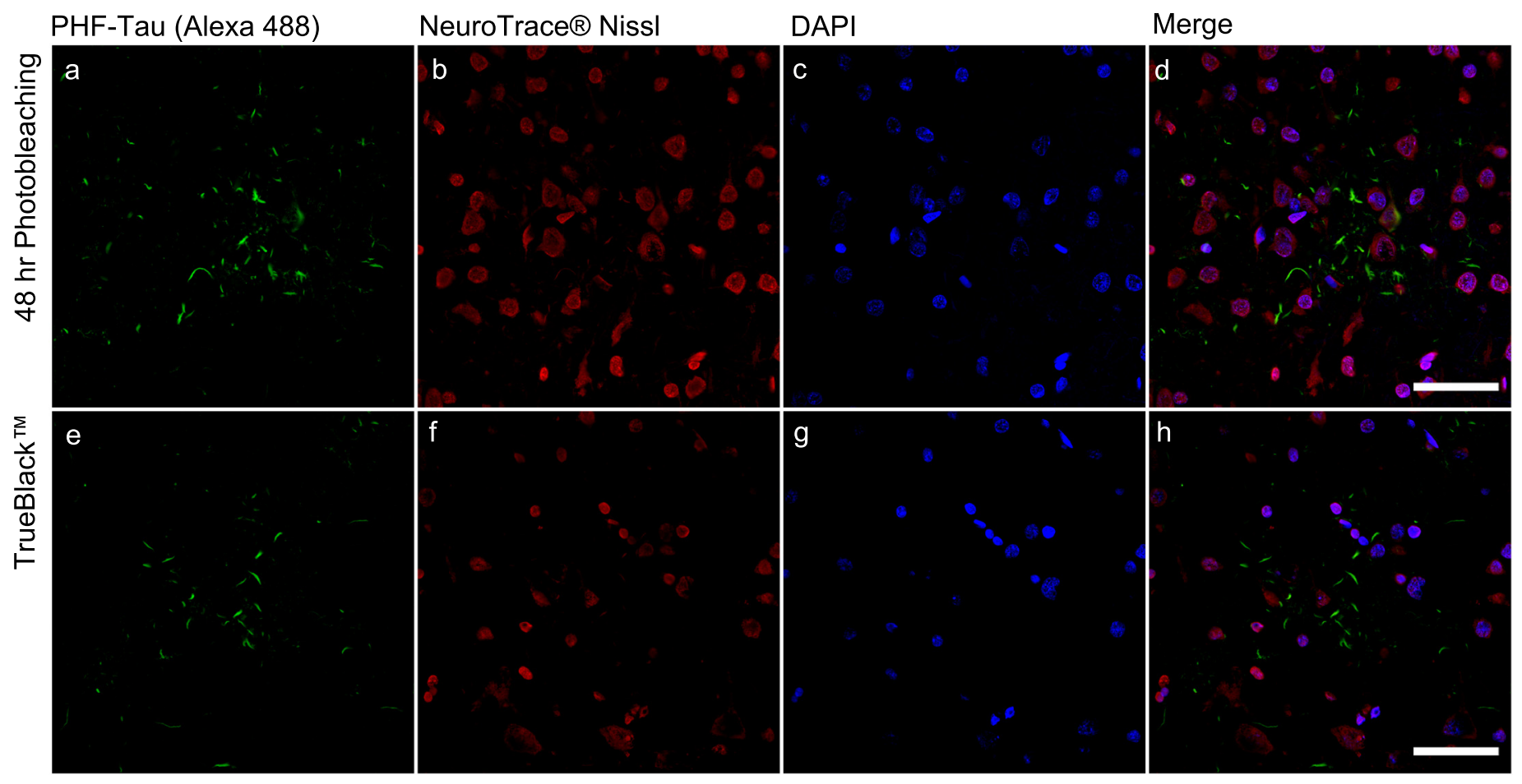

